\title{
Evaluación de los servicios ecosistémicos en la ciudad no planificada: El caso de Quetzaltenango
}

\author{
Mario Joaquín López Huertas
}

Artículo

\section{Afiliaciones:}

Universidad de San Carlos de Guatemala.

Fundación Hábitat para la Humanidad. Guatemala.

E-mail: maquinjo@gmail.com

Recibido: 16 de agosto del 2019

Aceptado: 7 de noviembre del 2019

\section{Mario Joaquín López Huertas} Arquitecto del Paisaje por la Universidad de Valparaíso en Chile, es profesor asistente de la División de Ingeniería de la Universidad de San Carlos en cátedras de Urbanismo, Gestión de Riesgos y Ecología. Es Coordinador de Proyectos en Hábitat para la Humanidad enfocado al desarrollo comunitario de poblaciones indígenas rurales.

\section{Resumen}

El crecimiento balanceado entre el medio natural y el medio construido es crucial para el sostenimiento de una sociedad. Con el aumento anual de la tasa de urbanización en la mayoría de los centros poblados a nivel mundial, se vuelve imprescindible considerar la conservación de los ecosistemas que proveen una cantidad de servicios para el desarrollo de las ciudades, los cuales pueden ser valorados desde los procesos económicos como pérdida o ganancia, hasta la percepción del bienestar de sus habitantes. Esta investigación realiza una evaluación de 7 Servicios Ecosistémicos (SE) en la ciudad de Quetzaltenango y analiza el consumo de una ciudad con crecimiento urbano desordenado. La falta de conocimiento, priorización, disponibilidad de suelo, condiciones económicas, aplicación correcta de instrumentos de planificación regional y del paisaje son los factores principales que no permiten considerar a los SE en la toma de decisiones sobre el territorio, generando un deterioro del medio natural que es representado en pérdidas económicas anuales. El estudio también presenta escenarios hipotéticos de recuperación ecosistémica para encaminar el desarrollo de la ciudad hacia una simbiosis natural-cultural.

Palabras clave: ecosistemas urbanos; planificación regional; planificación territorial; servicios ecosistémicos.

Evaluation of ecosystemic services in an unplanned city: The Quetzaltenango case

\begin{abstract}
The balanced growth between the natural and built environment is crucial for the sustainability of a society. With the current scenarios of the urban growth rate increasing in most of the cities around the world, the development of ecosystems that provide services to support cities becomes necessary. These services can range from economic processes with losses or profits to the perception of an inhabitant's wellbeing. This research project assesses seven ecosystem services (ES) in the city of Quetzaltenango and analyzes the consumption of unplanned urban growth. The lack of knowledge, prioritization, land access, economic conditions, and adequate governance of regional and landscape planning are the main factors that those who are involved in making decisions regarding land use do not give importance to the ES. As a result, these actions produce natural environment deterioration represented by annual economic losses. In addition, this study presents hypothetical scenarios for an ecosystem recovery, leading urban development towards a natural-cultural symbiosis.
\end{abstract}

Keywords: ecosystem services; land use planning; regional planning; urban ecosystems. 


\section{Introducción}

na de las razones más comunes por las que las ciudades se fundan sobre un territorio está relacionada con las condiciones que ofrece el medio natural para permitir el desarrollo cultural (Mumford, 1961). Conforme la ciudad va creciendo, densificando y desarrollando el espacio construido, el medio natural se fragmenta o desplaza hacia la periferia, reduciendo la capacidad de soporte para brindar los servicios que en un principio fueron la razón para la fundación de esa ciudad. Según las Naciones Unidas, se espera que para el 2050 más del $68 \%$ de la población mundial viva en zonas urbanas (2018). Para ese año, la ciudad de Quetzaltenango, que actualmente cuenta con 175,000 personas en la zona urbana y 210,000 en el área metropolitana (en conjunto con La Esperanza, San Mateo y Salcajá), alcanzará el millón de habitantes (BID, 2014).

La expansión urbana de la ciudad en las últimas décadas ha sido hacia zonas agrícolas y boscosas que, sin políticas ni instrumentos de ordenamiento territorial efectivos, generan una expansión sin control, deteriorando el medio natural y sus ecosistemas (Morán, 2018). Para sostener a una ciudad con este crecimiento, se debe cubrir la demanda en producción alimenticia, generación energética, provisión de agua, tratamiento de aguas residuales, gestión de residuos sólidos, capacidad de filtración de las emisiones de gases de efecto invernadero y disponibilidad de suelo (para vivienda, infraestructura y servicios), entre otros. Sin embargo, el Valle de Quetzaltenango presenta restricciones geográficas de un paisaje montañoso volcánico entre las microcuencas del Río Seco y Xequijel (cuenca del Río Samalá), lo que obliga a planificar con base en recursos finitos y a un metabolismo urbano (Rogers \& Gumuchdjian, 2000), de manera que el medio construido mantenga una relación estable y simbiótica con el medio natural (López Carrasco \& Valenzuela, 2017; Margulis, 2002).

Los Servicios Ecosistémicos (SE) se derivan de las características ecológicas, funciones y procesos de los ecosistemas presentes en un área territorial habitada que contribuyen de manera directa o indirecta al bienestar humano (Constanza et al., 1997). Estos deben estar vinculados de manera intrínseca a la ciudad dentro de la planificación regional, urbana y del paisaje. Se clasifican en servicios de aprovisionamiento, regulación, culturales y de apoyo (MEA, 2005), por lo que es necesario estudiarlos y socializar su importancia para que la sociedad y los tomadores de decisiones los integren dentro del manejo de recursos naturales y del territorio. Sin embargo, la valoración de los SE presenta una serie de desafíos como el hecho de que son heterogéneos al estar relacionados a una zona definida en proporción al espacio ocupado, generando problemas para definir su valor exacto sin que existan mercados para estos servicios, como la protección del riesgo a desastres, disminución del ruido y regulación climática, entre otros.

La evaluación de los SE a nivel de América Latina se ha desarrollado en su mayoría a escala regional más que urbana. Carreño, Frank \& Viglizzo (2012) evaluaron durante 50 años el intercambio entre la provisión de ecosistemas y servicios económicos (producción de fibras y alimentos de cultivos) en Argentina. Ricaurte et al. (2017) realizaron un mapeo de 19 tipos de humedales en Colombia con SIG participativo (Sistemas de Información Geográfica), permitiendo cuantificar la pérdida de los SE ante los cambios de uso de suelo. En Chile se evaluó la importancia en SE del bosque nativo versus las plantaciones forestales en la reducción de precipitación pluvial, aumento de escorrentía y reducción de la biota marina (Lara et al., 2009). En Perú, Baral et al. (2016) elaboraron un modelo de evaluación de los SE en plantaciones forestales para ser replicado en zonas con diferentes usos de suelo y los criterios para los tipos de paisaje. La deforestación para el uso agrícola en México fue analizada en costobeneficio, demostrando que los servicios de regulación (captura de carbono, regulación del ruido, regulación del clima, hábitat y refugio) son más importantes que los servicios de aprovisionamiento (producción alimenticia) (Lara-Pulido et al., 2018). Asimismo, Costa Rica ha demostrado que es posible el pago de SE y el reconocimiento de los mismos en países en desarrollo (Pagiola, 2008), donde Guatemala también presenta casos de estudio con experiencias en caza deportiva y conservación de la biodiversidad en cuencas hidrográficas (Ingram et al., 2014; Pagiola et al. 2010). 
En contextos urbanos, aunque fuera de Latinoamérica, se han realizado estudios de evaluación y valoración, determinando la existencia de SE con base a la composición de la ciudad (Bolund \& Hunhammar, 1999; Cortinovis \& Geneletti, 2018; Elmqvist et al., 2015), disponibilidad de espacios verdes (Thompson et al., 2012), impermeabilización del suelo (Szota et al., 2019), oscilación térmica, (Yang et al., 2016), almacenamiento de carbono (Svensson \& Eliasson, 1997), regulación del clima (Hough, 2004), o percepción del bienestar (El-Baghdadi \& Desha, 2016). En su mayoría, estos estudios se enfocan en la evaluación de los SE con la idea de proveer herramientas a los tomadores de decisiones para el desarrollo de políticas de uso de suelo enfocado al sostenimiento de los asentamientos humanos y del medio natural.

Esta investigación, se enfocó en la evaluación de 7 SE para la ciudad de Quetzaltenango con el objetivo de entender su deterioro y los efectos del crecimiento desordenado de una metrópoli que ha enfrentado dificultades para la implementación de instrumentos de planificación urbana, territorial, regional y del paisaje. La valoración, en algunos casos como pérdida o ganancia, permite una concientización sobre la importancia de los SE y los coloca como una prioridad ante la toma de decisiones para el desarrollo de la ciudad, la recuperación de ecosistemas urbanos, la reducción del riesgo a desastres y la adaptación al cambio climático.

\section{Metodología}

Según Constanza et al. (2017), no existe una manera específica para evaluar los SE. Sin embargo, el conocimiento de los mismos y los análisis del impacto económico que generan los SE a través del costo monetario representa una forma de reconocimiento para los tomadores de decisiones y la sociedad en general (Constanza et al., 2014; García et al., 2016). Son diversas las tipologías para la clasificación y definición de los SE (Haines-Young \& Potschin, 2018; MEA, 2005; TEEB, 2010), en este estudio, se eligió el modelo de Constanza et al. (1997), pues contiene servicios relevantes para la ciudad de Quetzaltenango, como el tratamiento de desechos sólidos, regulación ante inundaciones, hábitat y refugio. No se consideró el listado completo del autor citado (17), debido a la falta de disponibilidad de información local y que la evaluación de algunos SE requiere de un estudio exhaustivo en campos específicos. Además, algunos servicios no responden a las condiciones urbanas de Quetzaltenango como producción alimentaria, materias primas y formación de suelo. La Tabla 1 presenta el listado de los SE considerados en este estudio, el tipo de indicador, forma de evaluación y unidad de medida.

Tabla 1. Servicios Ecosistémicos Evaluados. Fuente: Elaboración propia con base a Constanza et al., 1997.

Fuente: Elaboración propia.

\begin{tabular}{|c|c|c|c|}
\hline $\begin{array}{l}\text { Servicio } \\
\text { Ecosistémico }\end{array}$ & Indicador & Evaluación & Medida \\
\hline $\begin{array}{l}\text { Captura y } \\
\text { Almacenamiento de } \\
\text { Carbono }\end{array}$ & $\begin{array}{l}\text { Cantidad de espacios verdes } \\
\text { y coberturas boscosas para la } \\
\text { absorción de GEl }\end{array}$ & $\begin{array}{l}\text { Calidad del aire } \\
\text { por emisiones } \\
\text { absorbidas }\end{array}$ & Tonelada/año \\
\hline Reducción del ruido & $\begin{array}{l}\text { Áreas boscosas como barreras del } \\
\text { ruido }\end{array}$ & $\begin{array}{l}\text { Calidad ambiental } \\
\text { por el ruido } \\
\text { disminuido }\end{array}$ & Decibeles/día \\
\hline $\begin{array}{l}\text { Regulación de Micro- } \\
\text { clima y efecto de } \\
\text { Enfriamiento }\end{array}$ & $\begin{array}{l}\text { Areas boscosas para control de } \\
\text { temperatura }\end{array}$ & $\begin{array}{l}\text { Gasto en sistemas } \\
\text { alternativos de } \\
\text { enfriamiento }\end{array}$ & ${ }^{\circ} \mathrm{C}$ \\
\hline $\begin{array}{l}\text { Tratamiento de } \\
\text { Aguas Residuales }\end{array}$ & $\begin{array}{l}\text { Volumen de agua tratada en } \\
\text { humedales, pantanos, etc. }\end{array}$ & $\begin{array}{l}\text { Pago en } \\
\text { Quetzales (Q.) de } \\
\text { aqua tratada }\end{array}$ & $\begin{array}{l}\text { Metros } \\
\text { cúbicos/año }\end{array}$ \\
\hline $\begin{array}{l}\text { Tratamiento de } \\
\text { Desechos Sólidos }\end{array}$ & Cantidad de desechos reciclados & productos & $\begin{array}{l}\text { Kilogramos/ } \\
\text { año }\end{array}$ \\
\hline Hábitat y Refugio & $\begin{array}{l}\text { \% del área urbana ocupada por } \\
\text { ecosistemas }\end{array}$ & $\begin{array}{l}\text { Servicios en Q. } \\
\text { para la ciudad }\end{array}$ & Hectáreas/año \\
\hline Recreación & $\begin{array}{l}\text { \# de personas que utilizan } \\
\text { espacios verdes para su } \\
\text { recreación }\end{array}$ & $\begin{array}{l}\text { Inversión en Q. } \\
\text { para recreación }\end{array}$ & Visitas/año \\
\hline
\end{tabular}


El tipo de evaluación de cada SE fue elaborada con base en sus condiciones locales y la capacidad para ser percibidos por la población. De ahí se consideran dos tipos de evaluación: la primera es la calidad ambiental a través de los SE de captura y almacenamiento del carbono, reducción del ruido y regulación del microclima. Su impacto repercute en la calidad ambiental de la ciudad y pueden ser percibidos en toda su extensión, por lo que no requieren una aproximación de valor económico. La segunda evaluación se escogió para los SE de tratamiento de aguas residuales, tratamiento de desechos sólidos, hábitat, refugio y recreación, que representan servicios que se perciben menos dentro de la población o culturalmente no existen, por ejemplo, áreas de impacto definidas para disponer los desechos sólidos y líquidos a donde no todos tienen acceso o desconocen del tipo de tratamiento, así como puntos de la ciudad que permiten algún tipo de recreación, promoción de la biodiversidad y reducción del riesgo a desastres inexistentes o sin una distribución equitativa en la superficie urbana. Por lo anterior, se ve en la necesidad de generar una aproximación económica para cuatro SE que ponga en valor su relevancia ante el funcionamiento de la ciudad y sea de fácil entendimiento para la población en general.

\section{Captura y Almacenamiento de Carbono}

La calidad del aire de una ciudad repercute en la salud de sus habitantes. Mientras mayor sean las áreas verdes y las coberturas boscosas, mayor será la captura y almacenamiento de carbono en la ciudad, proporcionado a las emisiones de gases de efecto invernadero (Svensson \& Eliasson, 1997). La eficiencia de la captura dependerá tanto de la variedad de especies boscosas como de su estructura y edad. A mayor diversidad, mayor eficiencia en el almacenamiento de carbono. Como referencia, 1 hectárea de bosque mixto puede absorber 15 toneladas de partículas al año, mientras que un bosque de la familia Pinaceae (por ejemplo, la endémica Abies Guatemalensis) absorbe de dos a tres veces más (Beier \& Gundersen, 1989).

Quetzaltenango cuenta con 293 hectáreas de áreas verdes (BID, 2014), que incluye el parque Regional Municipal situado en la zona sur del municipio en el área rural del Palajunoj. Este parque no incide en la absorción por la producción de gases del área urbana. A efectos de esta medición, se consideraron las áreas verdes a las que la población tiene acceso dentro de la ciudad. El dato se calculó con la plataforma ArqGIS y SAS Planet en su última actualización para el 2018, resultando un total de 23.62 hectáreas.

Para la evaluación del SE, se estimó la calidad del aire entre la producción y absorción de gases dentro de la ciudad. Por una parte, se cuantificaron las emisiones de gases de efecto invernadero del transporte terrestre de personas y mercadería, que es uno de los problemas más evidentes en la ciudad de Quetzaltenango y nos permite tener una medida en toneladas de dióxido de Carbono (CO2). El parque vehicular de la ciudad oscila entre 257,336 vehículos, según el registro de SAT (2019), con un promedio de emisiones de un "típico vehículo de pasajeros", según la EPA (2018), de 4.6 toneladas métricas. Para realizar el balance entre la producción y absorción se generó la ecuación 1:
(1) $A b s=B X C c$
$E m s=V X E m s P$
$C A=A b s-E m s$
Donde:

Abs: Absorciones de $\mathrm{CO} 2$

$B$ : Bosque en áreas verdes medido en hectáreas

Cc: Capacidad de captura del bosque en toneladas por año

Ems: Emisiones de $\mathrm{CO} 2$

$V$ : número de vehículos

EmsP: Emisiones promedio de los vehículos

CA: Calidad del aire 


\section{Reducción del Ruido}

La condición de un ecosistema para lograr la reducción del ruido en la ciudad considera elementos muy puntuales tales como: área de estudio, el ruido generado por el tráfico y su dinámica con la morfología de las vías, las actividades humanas en el espacio público, la distancia de la emisión con respecto a la recepción (una distancia duplicada de la fuente reduce hasta $3 \mathrm{~dB}$ ), las condiciones del suelo (áreas verdes o suelo sin pavimentar disminuye hasta $3 \mathrm{~dB}$ ) y en algunos casos, elementos de propagación. Bolund \& Hunhammar (1999) postulan que el ruido aumenta cuando existe una superficie de agua cercana a la fuente de emisión.

La densidad de doseles arbóreos presentes en las áreas verdes contribuye a la disminución de la intensidad del ruido a distancias lejanas, mientras que los troncos de los árboles reducen la intensidad a distancias cortas. Sin embargo, la capacidad de reducción dependerá de la composición de la cobertura boscosa, que debe ser heterogénea y con medias mínimas de $20 \mathrm{~m}$ de ancho y $14 \mathrm{~m}$ de alto con follaje denso y perenne (Posada, Arroyave \& Fernández, 2009).

La medición de la contaminación auditiva se realizó por medio de la aplicación sonómetro (Sound Meter) para dispositivos Android y Sound Level Analyzer Life para sistema iOS. Se realizaron recorridos en 12 puntos de la ciudad para obtener una variación según la densidad poblacional definida por el Plan de Ordenamiento Terrritorial (Municipalidad de Quetzaltenango, 2017). Cada recorrido tuvo una extensión promedio de $3 \mathrm{~km}$ con 10 lecturas a cada 300 metros. Todos los datos de campo se recolectaron entre las 12:00 y las 14:00, entre los días lunes y viernes por ser los momentos de mayor afluencia vehicular y actividades humanas.

\section{Regulación de micro-clima y efecto de enfriamiento}

La modificación que sufre el territorio donde se asienta la ciudad tiene repercusiones sobre sus dinámicas térmicas (Carreras et al., 1990). El crecimiento y la densificación incrementa el área construida que, ante la desproporción de áreas que absorban el calor (cuerpos de agua y áreas verdes con doseles arbóreos medios y altos) producido por las actividades de la ciudad y la radiación solar, genera un microclima con una temperatura mayor con respecto a la que se encuentra en la periferia, generando el efecto conocido como la Isla de Calor (Yang et al., 2016). El efecto eleva la temperatura no sólo por la carencia de espacios verdes y azules, sino por superficies de materiales que absorben la energía solar (concreto, asfalto) y lo refractan hacia el espacio público.

Mientras mayor sea el espacio de los ecosistemas en zonas urbanas, mayor será la capacidad para regular el clima y los efectos de la isla urbana. El bosque nativo tiene la competencia para interceptar la luz del sol (en promedio $1000 \mathrm{mj}$ por árbol/día), absorber la lluvia y liberar hasta 450 litros en humedad al aire, generando un microclima por debajo de su sombra que es menos cambiante que en las áreas construidas (Hough, 2004). La regulación climática a través de ecosistemas permite una reducción en la inversión de sistemas de enfriamiento, calefacción en viviendas y edificios, brindando un servicio de bajo costo y larga duración para sus usuarios (Yeang, 2002).

Para la medición del SE, fueron planteados 6 recorridos que variaron desde 6 hasta $10 \mathrm{~km}$, para abarcar desde los extremos de la ciudad hacia las zonas más densas. Los puntos de partida fueron parques o áreas verdes (Colonia Molina, Los Trigales, Ciudad de Los Altos, Colonia El Maestro, Las Rosas y Salida a Almolonga), cruzando las avenidas más concurridas hasta llegar al centro. La herramienta de medición fue un Termómetro Ambiental Digital, tomando lecturas a cada 500 metros en el lapso de las 12:00 a las 14:00 horas, entre los días lunes y viernes por ser los momentos de mayor afluencia vehicular y actividades humanas. 


\section{Tratamiento de Aguas Residuales}

El Acuerdo Gubernativo 236-2006 del Congreso de Guatemala establece criterios y requisitos a cumplir para proteger los receptores de aguas residuales a través de un tratamiento adecuado. Actualmente, Quetzaltenango no cuenta con una cobertura municipal de plantas de tratamiento para las aguas residuales. La producción diaria en la ciudad es de 67,086.14 metros cúbicos, según la Dirección de Drenajes de Quetzaltenango. No existe una clasificación entre las aguas grises, negras y pluviales, ni el tipo de disposición de cada una, sino que las tres culminan en el caudal del Río Seco que se integra al Río Xequijel como subcuenca del Río Samalá. Esta falta de tratamiento del agua se considera, según el Acuerdo Gubernativo, una ilegalidad, además del deterioro de la calidad ecosistémica de una cuenca que desemboca en el mar.

Tradicionalmente, este SE es evaluado por la capacidad que tiene un ecosistema en tratar las aguas residuales y mantener la disponibilidad de agua en el sector, que en la mayoría de veces se realiza con la implementación y desarrollo de humedales que actúan como sedimentadores, retenedores de nitrógeno y fósforo, y reciclaje de nutrientes. El humedal brinda las condiciones para la biodiversidad y trabaja en el control de las inundaciones (Ewel, 1997). Sin embargo, en Quetzaltenango, la recuperación del agua consumida a través de un tratamiento no representa una prioridad debido a que la ciudad cuenta con una alta oferta de agua potable (Tacam, 2011), volviendo los esfuerzos para el tratamiento algo prescindible.

La evaluación del SE fue aproximada desde un escenario hipotético, donde la cuenca como un sistema de recolección no existe y la disponibilidad del agua es limitada. La ciudad tendría que invertir en un sistema de tratamiento de aguas residuales como única opción para garantizar el retorno del vital líquido a sus habitantes. Si bien el municipio no deroga un impuesto por la recolección y tratamiento de las aguas residuales, esa condición sería modificada ante la implementación de la infraestructura que llevaría el servicio hacia cada hogar.

Para determinar el costo por el tratamiento, se tomó a Chile como ejemplo, que a nivel Latinoamérica tiene la mayor cantidad de hogares conectados a un sistema de saneamiento (Reynolds, 2002), con una estimación de Q6.24/m3 al mes por recolección y tratamiento. Para el cálculo del SE, fue planteada la ecuación 2:

(2) $C t a t=C u \times D c \times T e$

Donde:

Ctat: costo total anual del tratamiento

Cu: costo unitario por tratamiento y recolección (Quetzales/m3)

Dc: descarga de la ciudad (m3/día)

Te: Tiempo de estimación (1 año)

\section{Tratamiento de Desechos Sólidos y Reciclaje de Nutrientes}

En la actualidad, la generación de desechos sólidos en la ciudad es de 225 toneladas en promedio al día (USAID, 2017). Está conformada por $60 \%$ de materia orgánica y $40 \%$ inorgánica, la cual se vierte en un botadero municipal situado a 14 kilómetros del centro, en el área de Xepache. En el botadero no se practica la clasificación de desechos ni se da el tratamiento adecuado para la conformación de un relleno sanitario degradando el suelo y el entorno, provocando lixiviados que ponen en riesgo la calidad de los acuíferos cercanos.

EI SE está relacionado a los procesos de biodegradación de los desechos sólidos producidos por una población determinada. Su trabajo es reciclar nutrientes para integrarse al suelo, brindando un beneficio en términos de salud y disponibilidad de suelo fértil (Adedipe et al., 2005). Para la evaluación del SE, se utilizó una aproximación cuantitativa de los ingresos que se pierden al no aprovechar los desechos a través del compostaje y reciclaje de los mismos. Entre los materiales identificados, se consideró el $60 \%$ de materia orgánica, $14 \%$ de papel y $5 \%$ vidrio, como resultado a la consulta 
en la Dirección de Servicios Ambientales de la Municipalidad de Quetzaltenango (DSA). A cada material, se le asignó el porcentaje del total que puede ser reciclado. Se consultaron a recicladoras locales y nacionales sobre los costos del material por kg para determinar un total por día y por año.

\section{Hábitat y Refugio}

El territorio de Quetzaltenango está inmerso dentro del ecosistema Bosque húmedo montano bajo tropical, una zona de vida que presenta una interacción entre la evapotranspiración potencial y precipitación pluvial de $0.67 \mathrm{~mm}$ que propicia excedentes de agua (IARNA, 2018), permitiendo las vertientes que forman la microcuenca del Río Seco, como parte del sistema entrelazado y cambiante de la Cuenca del Río Samalá. Este es uno de los sistemas fluviales del Holoceno más grandes en el suroccidente guatemalteco (Viera, 2003).

El bosque de Quetzaltenango ha sido fragmentado con el crecimiento demográfico y desplazado hacia la periferia, donde también se ha reducido debido a la tala incontrolable de árboles y la actividad minera. Las áreas verdes presentes en la ciudad no consolidan un corredor biológico ni albergan especies arbóreas nativas que permitan un desarrollo del ecosistema terrestre (Ruiz, Cardona \& Duque, 2012). Por otro lado, el ecosistema fluvial se ha degradado al ser utilizado como desfogue de las aguas residuales que produce diariamente la ciudad sin recibir ningún tratamiento. Esta contaminación repercute en toda el área hidrográfica del Samalá, incluso en los ecosistemas marinos donde esta desemboca (Escobar, 2002).

El SE Hábitat y Refugio evalúa la conectividad ecológica entre el bosque, áreas verdes urbanas o ecosistemas fluviales, al igual que la complejidad en la estructura y cantidad de espacio que ocupa el ecosistema dentro de la ciudad. Mientras mayor sea la cobertura, mayor será el beneficio. Para evaluarlo, se desarrolló un escenario hipotético de recuperación de los 3 ecosistemas que caracterizan el paisaje del Valle de Quetzaltenango y que han sido deteriorados por el avance de la huella urbana. A cada uno, se le asignó el valor que, según Constanza et al. (2014), brindan los ecosistemas para el sostenimiento de actividades humanas por estructura y composición. El dato permite monetizar la pérdida ante el deterioro del medio natural.

\section{Recreación}

Las ciudades densamente pobladas con sus actividades diarias pueden generar un ambiente hostil y estresante para sus habitantes (Mumford, 1961). Los ecosistemas urbanos que brindan espacios para recreación y esparcimiento son los más valorados dentro de los SE en las ciudades (Bolund \& Hunhammar, 1999) y proveen aspectos estéticos que son de fácil percepción para sus habitantes, lo que genera una mayor identificación del beneficio. Las áreas de recreación urbana pueden ser una fuente de ingresos para el municipio y de generación de actividades económicas dentro del mismo. También existen beneficios psicológicos dados por la relación entre los humanos y el medio natural a través de la biofilia, que postula que el contacto con la naturaleza eleva y mantiene el estándar de vida de la población (El-Baghdadi \& Desha, 2016; Russo \& Cirella, 2017). El estudio de Thompson et al. (2012) es un ejemplo que evalúa cómo los niveles de estrés en áreas urbanas desfavorecidas actúan sobre el nivel de bienestar de la población.

Para evaluar el SE, fue empleada la metodología de Castillo et al. (2013) en su estudio por medio de encuestas para medir el conocimiento de los ciudadanos en los Servicios Ecosistémicos Urbanos de Quetzaltenango y Antigua Guatemala. En este caso, la encuesta es una caracterización de percepciones para identificar las preferencias de recreación en la población de Quetzaltenango. El instrumento mide el conocimiento de las áreas verdes, tiempo de permanencia, disponibilidad de pago para la recreación, factores que influyen en la visita y preferencia de zonas recreativas, entre otros. Además, se identificó el costo invertido para la recreación dentro de los centros comerciales como un sustituto ante la carencia de áreas verdes y que responde a una actividad 
de auge en Quetzaltenango. El costo se evaluó como una oportunidad de ingreso económico a los programas de conservación y desarrollo de parques y áreas verdes en la ciudad. Se consideró una muestra de 150 encuestados divididos por igual en 3 zonas de la ciudad: Zona 10 (espacio transitorio entre la zona urbana y rural), Zona 3 (área comercial más concurrida de la ciudad) y Zona 8 (área residencial con crecimiento urbano). La encuesta contiene un total de 9 preguntas.

\section{Resultados}

\section{Captura y Almacenamiento de Carbono}

El cálculo de la calidad del aire brinda los siguientes resultados:

Abs $=B(H a) X C$.$c (t/año) =23.62$ ha X 15 t/año $=354.3$ t/año

$E m s=V(\#) X E m s P(t / a n ̃ o)=257,336 \times 4.6$ t/año $=1,183,745.6$ t/año

$C A=A b s-E m s=354.3-1,183,745.6=-1,183,391.3$ t/año.

El SE es imperceptible debido a que se logra capturar solamente el $0.02 \%$ de las emisiones de CO2/año, las pocas áreas boscosas y verdes dentro de la ciudad no representan un volumen de absorción suficiente para la generación actual de CO2. Una mayor presencia del servicio se podría percibir con un aumento de las coberturas forestales, aunque la brecha para alcanzar las emisiones aún es excesiva. Por lo anterior, Quetzaltenango presenta un déficit en la captura de carbono.

\section{Reducción del Ruido}

Figura 1. Ruido versus densidad poblacional de Quetzaltenango. Fuente: Elaboración propia con base a documentos de la Municipalidad de Quetzaltenango (2017) y estudios de campo.
La Figura 1. Intervalos de intensidades en decibeles que se perciben en los puntos críticos.

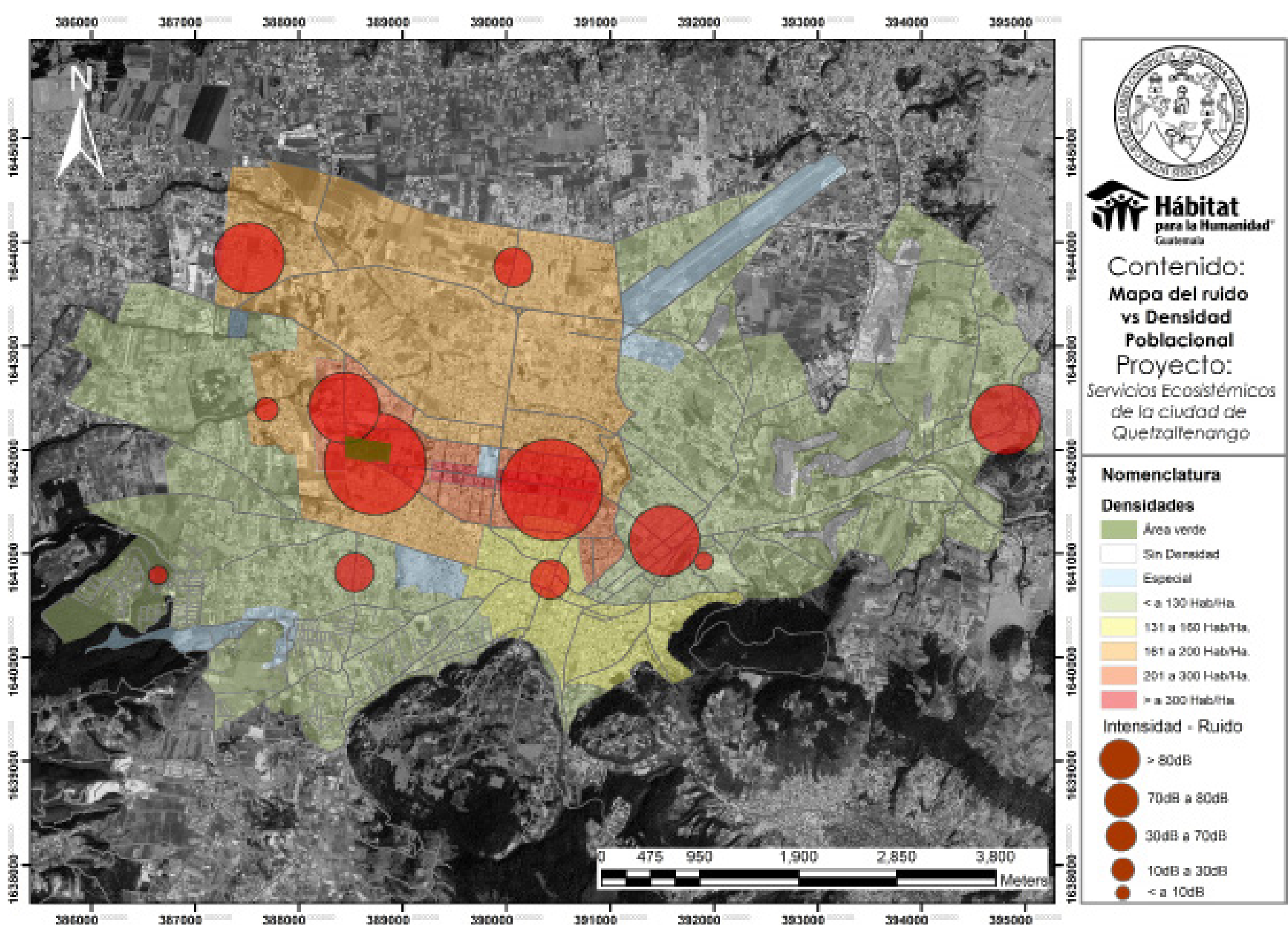


La lectura más alta está por encima de los $85 \mathrm{~dB}$ en las zonas más densas y avenidas concurridas (zona comercial y oficinas), superando el recomendado por la OMS (2015) en una exposición de 8 horas/día. Los siguientes intervalos se encuentran por debajo de la norma. Por su parte, el área verde que figura en el mapa es el Parque Zoológico Minerva como la única que cumple con el servicio ecosistémico de reducción del ruido. En el resto de la ciudad, los elementos que funcionan como barreras son construcciones de mampostería con diversos usos que reducen desde 10 hasta $15 \mathrm{~dB}$, pero requieren de una mayor inversión.

\section{Regulación de microclima y efecto de enfriamiento}

La Figura 2 muestra la zonificación sobre la oscilación térmica urbana en la ciudad de Quetzaltenango. En los extremos oeste y sur figura la menor temperatura por su cercanía a coberturas boscosas y caudales de río. Si bien la temperatura media registrada en Quetzaltenango es $14.0^{\circ} \mathrm{C}$ (INSIVUMEH, 2019), el trabajo de campo presentó una oscilación térmica entre $17^{\circ} \mathrm{C}$ hasta $31^{\circ} \mathrm{C}$. Vale la pena mencionar que, si bien existe una variación entre 170 a 200 metros de altitud en el relieve dentro de la zona urbana, esta no fue significativa en los resultados de las mediciones en campo.

El incremento de temperaturas se presenta en las zonas densificadas para usos comerciales y oficinas, así como en calles y avenidas donde transita la mayor cantidad de vehículos que emiten su propio calor por la combustión y el tráfico lento. Algunas áreas verdes mitigan el efecto térmico hacia el interior, aunque no son capaces de esparcir el microclima hacia las zonas adyacentes donde no existen coberturas boscosas y predomina el espacio construido.

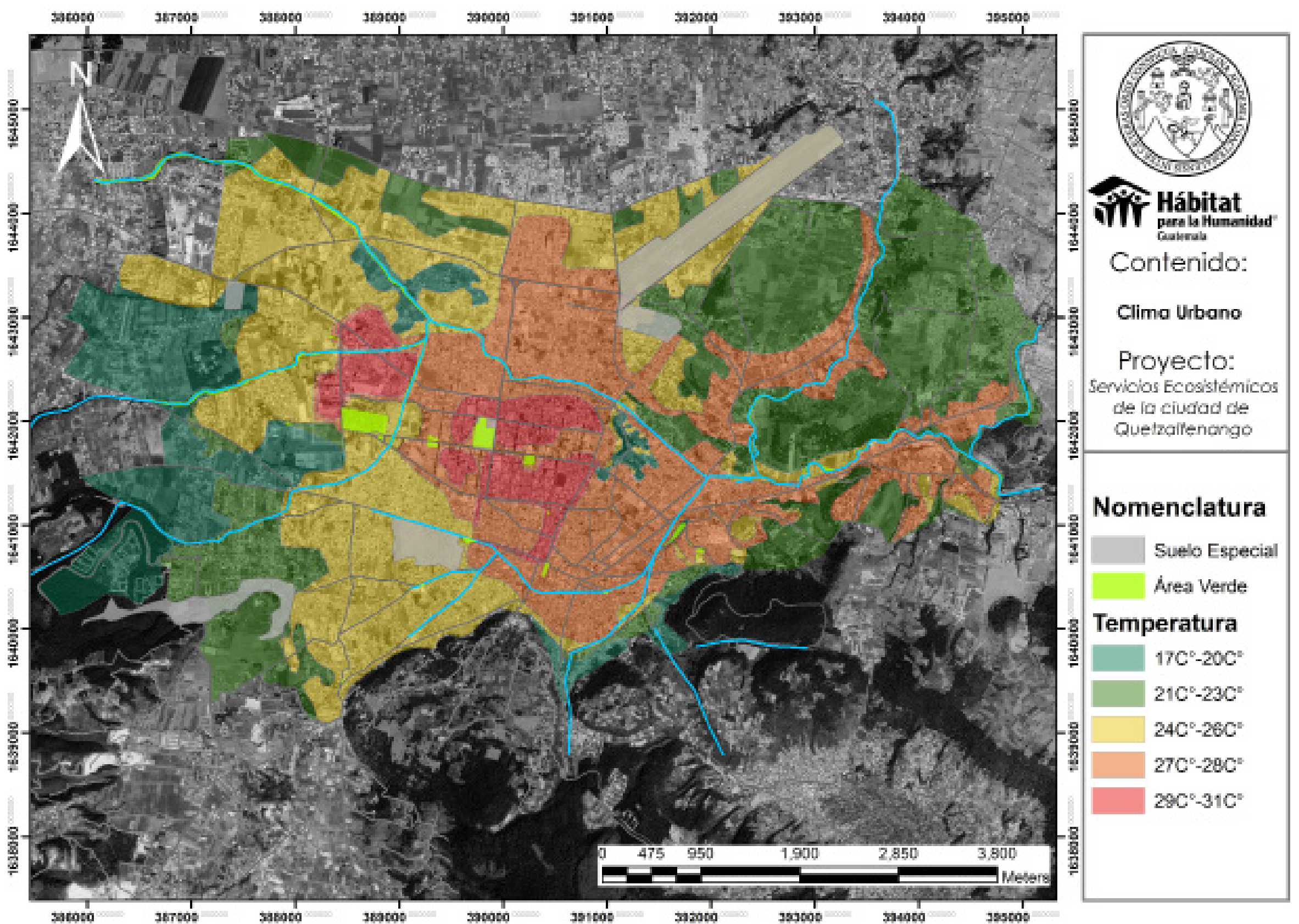

Figura 2. Temperatura urbana. Fuente: Elaboración propia con base a IGN (2017) y estudios de campo. 
Tabla 2. Posibles ingresos con el reciclaje de materia orgánica, papel y vidrio. Fuente: Elaboración propia con base en USAID (2017) y estudios de campo.
En lugares donde existe una permanencia igual o mayor de 8 horas diarias (vivienda o trabajo) y no existe una construcción bioclimática. El incremento de la temperatura incide en la compra y adquisición de servicios que reduzcan el efecto de la isla de calor, entre ellos, el aire acondicionado. Este tiene un costo de inversión inicial y mantenimiento anual cercano a Q12,000.00 para una superficie promedio de $50 \mathrm{~m} 2$, mientras que un árbol con follaje y altura sobre una estructura con el mismo metraje brindaría los mismos beneficios a un precio mucho menor (Akbari et al., 1997).

\section{Tratamiento de Aguas Residuales}

Considerando los costos con base a la producción real diaria de aguas residuales en un año se tuvieron los siguientes resultados:

Ctat $=$ Cu $\times$ Dc $\times$ Te $=$ Q6.24/m3 X67,086.14m3/día $\times 365$

Ctat $=\mathrm{Q} 152,795,392.00$

El dato representa una aproximación sobre el monto económico que la población de Quetzaltenango ahorra al utilizar la cuenca del Río Seco como sistema de recolección de aguas residuales sin darle algún tratamiento.

\section{Tratamiento de Desechos Sólidos y Reciclaje de Nutrientes}

La Tabla 2 presenta la aproximación cuantitativa total por día de los ingresos que generaría el reciclaje de la producción de basura de la ciudad. El material más valorado es la materia orgánica que se convierte en compost, debido al enfoque actual de agricultura orgánica que presenta mayor atracción para los mercados de exportación (Campos et al., 2017; Martínez, Oaxaca \& Guerra, 2011). En total, se cuantificó Q38,832,642.00 como un ingreso que puede establecerse para el municipio o a empresas privadas en caso de aprovechar los recursos y utilizar el servicio ecosistémico del tratamiento y reciclaje de nutrientes.

\section{POSIBLES INGRESOS CON EL RECICLAJE DE 225 t/día DE DESECHOS EN QUETZALTENANGO}

\begin{tabular}{|c|c|c|c|c|c|c|}
\hline $\begin{array}{l}\text { Tipo de } \\
\text { Material }\end{array}$ & $\begin{array}{l}\% \text { del } \\
\text { total }\end{array}$ & $\begin{array}{l}\text { Cantidad } \\
\mathrm{Kg}\end{array}$ & $\begin{array}{l}\% \text { a } \\
\text { reciclar }\end{array}$ & $\begin{array}{l}\text { Costo por } \\
\mathrm{Kg}\end{array}$ & $\begin{array}{l}\text { Valor por } \\
\text { día }\end{array}$ & Valor anual \\
\hline $\begin{array}{l}\text { Materia } \\
\text { Orgánica }\end{array}$ & $60 \%$ & 135000 & $50 \%$ & $\begin{array}{l}\mathrm{Q} \\
1.50\end{array}$ & $\begin{array}{l}Q \\
101,250.00\end{array}$ & $\begin{array}{l}Q \\
36,956,250.00\end{array}$ \\
\hline Papel & $14 \%$ & 31275 & $66 \%$ & $\begin{array}{c}Q \\
0.20\end{array}$ & $\begin{array}{l}Q \\
4,128.30\end{array}$ & $\begin{array}{l}Q \\
1,506,829.50\end{array}$ \\
\hline Vidrio & $5 \%$ & 11250 & $100 \%$ & $\begin{array}{c}Q \\
0.09\end{array}$ & $\begin{array}{l}Q \\
1,012.50\end{array}$ & $\begin{array}{l}Q \\
369,562.50\end{array}$ \\
\hline & & & & & TOTAL & $\begin{array}{l}Q \\
38,832,642.00\end{array}$ \\
\hline
\end{tabular}

Hábitat y Refugio

El bosque relicto podría ser la guía para vincular la sucesión secundaria sobre el avance de la frontera agrícola y la recuperación del suelo urbano para guiar la forma de la ciudad a través del medio natural (McHarg, 1967). Para el Río Seco y Xequijel, se debería de ampliar el ancho del caudal con vegetación nativa en los extremos para reducir la velocidad de la escorrentía y generar un sistema de filtración del agua (Szota et al., 2019). La recuperación del Pantano de la zona 2 , que tiene una ocupación completa por uso residencial, reduciría la incidencia de inundaciones (López, 2019), regulando el caudal de los ríos. Este es un sistema vivo de tratamiento de agua y un ecosistema que concentra alta biodiversidad (Elmqvist et al., 2015). La Tabla 3 presenta el valor de los tres ecosistemas según las hectáreas que ocupan el escenario hipotético de la Figura 3. 
Tabla 3 Valor de los ecosistemas en Ha./ año. Fuente: Elaboración propia con base en Constanza et al. (2014).

\section{VALOR DE LOS ECOSISTEMAS DE QUETZALTENANGO}

\begin{tabular}{|c|c|c|c|c|}
\hline Ecosistema & Cantidad en Ha. & Valor $\mathrm{Ha}$ & Año en $Q$ & Subtotal \\
\hline Río & 241 & Q & $32,429.20$ & Q $7,815,437.20$ \\
\hline Bosque & 650 & Q & $40,006.40$ & Q $26,004,160.00$ \\
\hline Pantano & 72 & $Q$ & $195,183.20$ & Q $14,053,190.40$ \\
\hline & & TOTAL & & Q $47,872,787.60$ \\
\hline
\end{tabular}

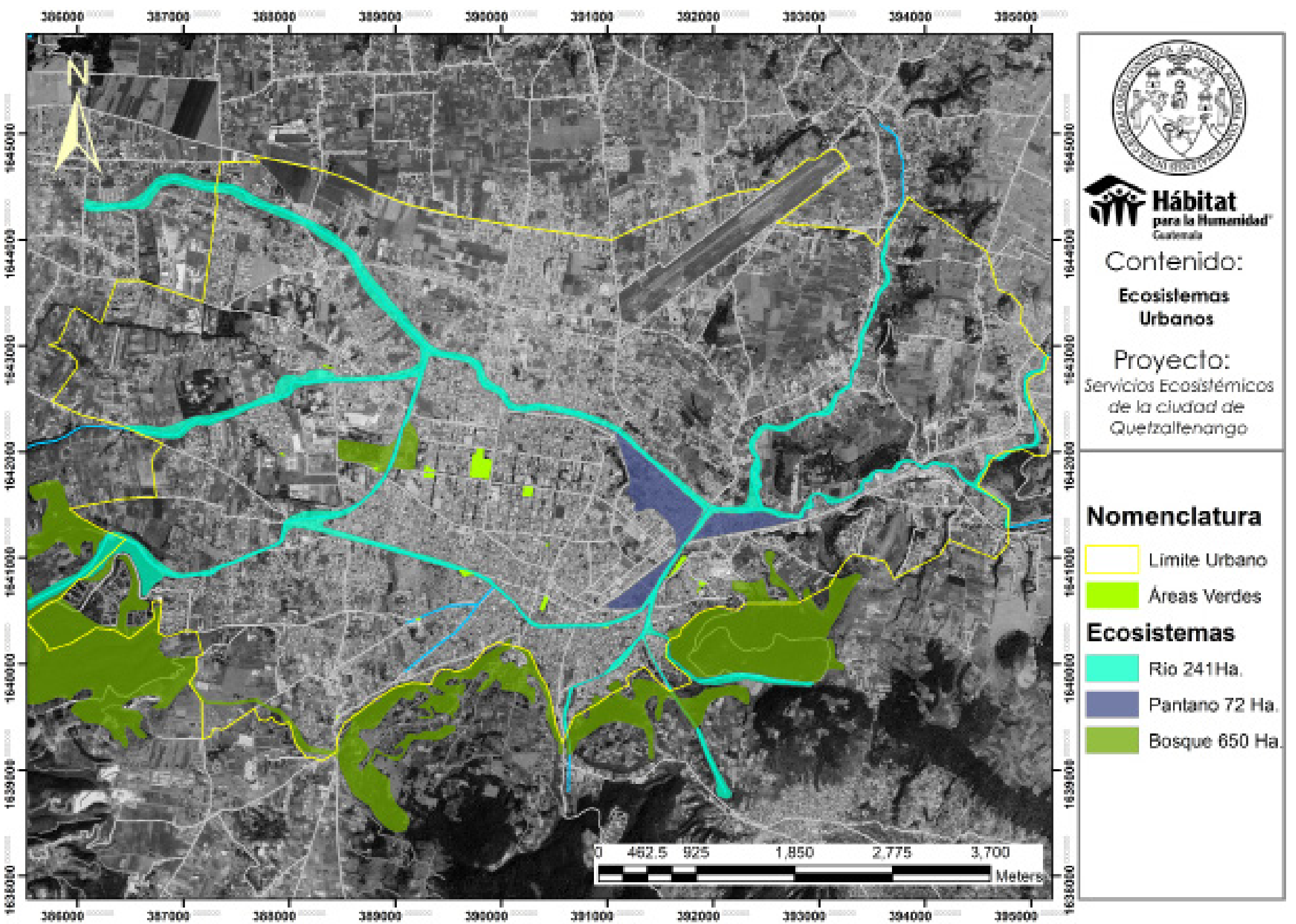

Figura 3. Recuperación de ecosistemas urbanos. Fuente: Elaboración propia con base en IGN (2017).
A El beneficio de recuperar y desarrollar estos tres ecosistemas es más directo a través del mercado donde se sitúa. En el caso del bosque, según el criterio de García et al. (2016), existen los productos forestales no maderables (PFNM), la leña de los árboles para combustible y madera para la construcción. La ciudad puede elevar su disponibilidad de agua al recuperar los caudales de los ríos y disminuir el uso de pozos que agoten los acuíferos, situación que generará un déficit a partir del 2030 e incrementará el precio al servicio del agua (Tacam, 2011). Los ecosistemas como el pantano representan una fortaleza para el medio construido a través de su contribución para la reducción del riesgo a desastres y la adaptación al cambio climático como Servicios de Regulación (Lo, 2016; Murti \& Buyck, 2014).

\section{Recreación}

En la Pregunta 1 (P1), sobre el conocimiento de las áreas verdes, los resultados resaltan que el $100 \%$ de la población conoce al menos el $75 \%$ de ellas. En la Pregunta 2 (P2), la Figura 4 muestra el tiempo que permanecen las personas cuando visitan las áreas verdes. Más del $45 \%$ de los entrevistados afirman que realizan una visita de 15 minutos, mientras que menos del $7 \%$ permanecen por 2 horas o más. Con respecto a la 
Figura 4. Tiempo de permanencia en áreas verdes.

Fuente: Elaboración propia con base en Castillo et al. (2013) y estudios de campo.
Figura 5. Factores que influyen en la poca visita a las áreas verdes. Fuente: Elaboración propia con base en Castillo et al. (2013) y estudios de campo.
Pregunta 3 (P3), sobre si estarían dispuestos a pagar para ingresar a las áreas verdes el $100 \%$ respondió que no.

\section{Cuando visita el área verde ¿Cuánto tiempo permanece en ella?}

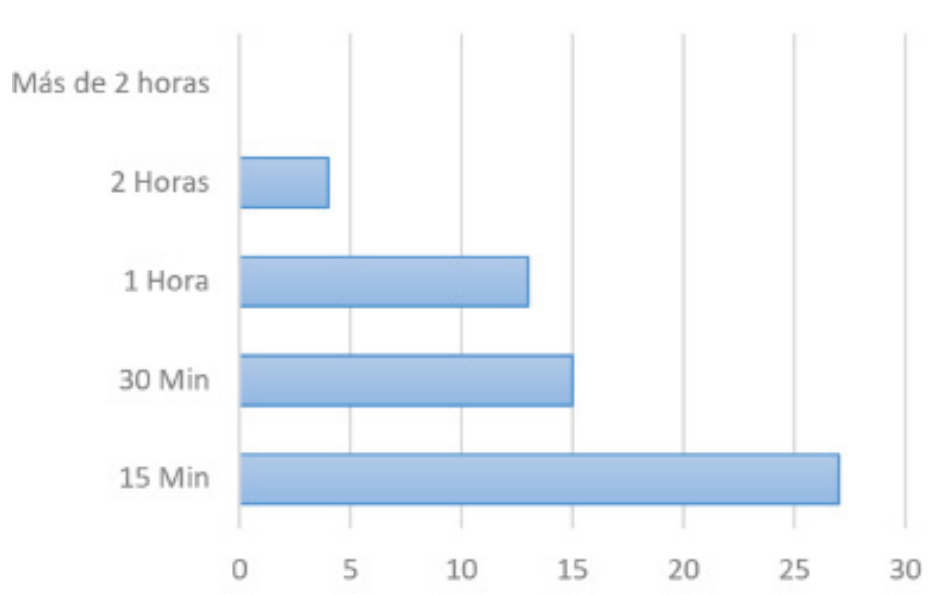

En los aspectos limitantes, se preguntó cómo se consideran los accesos a las áreas verdes (P4). El 17\% respondió que son de acceso difícil, el $77 \%$ de acceso medio y sólo el $6 \%$ dijo que son de fácil acceso.

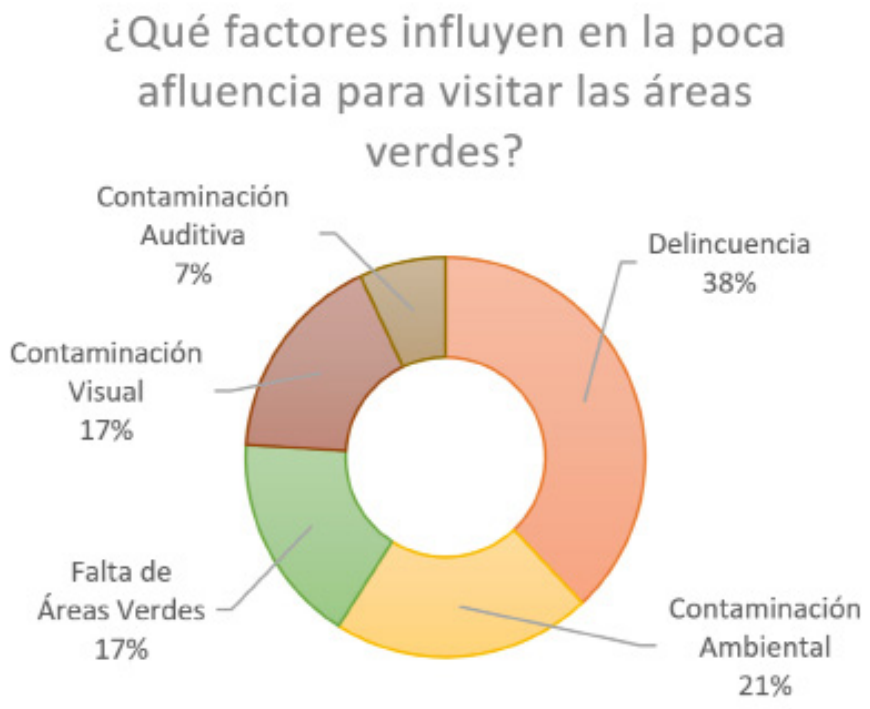

La Figura 5 presenta los factores que influyen en la poca afluencia para visitar las áreas verdes (P5). El mayor porcentaje se divide en problemas de contaminación, donde el $21 \%$ es ambiental, $17 \%$ visual y $7 \%$ auditiva. El $38 \%$ considera la delincuencia como una limitante y el $17 \%$ se debe a la falta de áreas verdes cercanas a las viviendas.

La tercera parte evaluó las preferencias de los habitantes quezaltecos. En la pregunta de qué lugares les parecen más atractivos (P6), el 44\% prefiere el Centro Histórico, el $38 \%$ los parques y áreas verdes y el $18 \%$ los centros comerciales. Con respecto a los lugares que generan mayor visita de turistas a la ciudad (P7), el $46 \%$ considera que es el Centro Histórico, $28 \%$ los centros comerciales y $26 \%$ perciben como atractivo los parques. La pregunta sobre los lugares que más frecuentan los entrevistados se presenta en la Figura 6, el $41 \%$ respondió que prefiere los centros comerciales, $36 \%$ las áreas verdes y el $23 \%$ el Centro Histórico, como una contrariedad de la Pregunta 6. En la consulta sobre los gastos por cada visita al centro comercial al mes, se definió un promedio de Q35.00 por persona.

\section{¿Qué lugares frecuenta de la}

ciudad para su recreación?

$\square$ Centro Histórico $\square$ Centros Comerciales $\square$ Parques

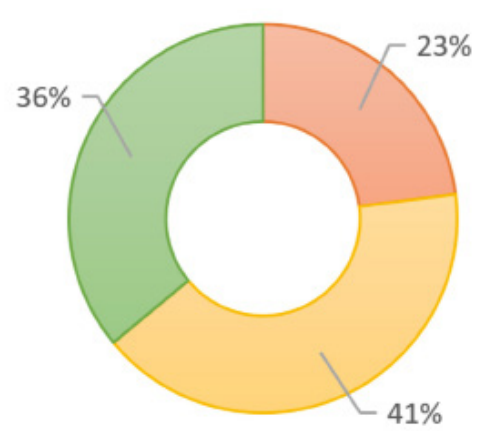


Considerando los resultados de la muestra, donde el $41 \%$ de la población de la ciudad visita los centros comerciales para recreación por la cantidad de habitantes $(175,000$ de la población Quetzaltenango x $0.41=153,801)$ por el gasto por persona $(\mathrm{Q} 35.00)$, es igual a Q26,484,532.00/mes, que supera el 100\% del presupuesto para la protección ambiental de la Municipalidad de Quetzaltenango (ordenación de desechos, reducción de la contaminación, protección de la diversidad biológica y del paisaje, investigación y desarrollo relacionado con la protección ambiental, lo que sería Q21,066,569 de presupuesto, según SIAF-MUNI, 2018).

\section{Discusión}

El proceso de evaluación diferenció a los SE de acuerdo con su rendimiento, cobertura, vida ecosistémica y percepción de importancia. La captura y almacenamiento de carbono, reducción del ruido y regulación de microclima y efecto de enfriamiento son tres servicios que presentan un déficit y un muy bajo rendimiento. A los tres se les puede atribuir una carente cobertura boscosa dentro del contexto urbano, aunque cada uno puede ser intervenido de distintas formas: la fuente de gases de efecto invernadero en la ciudad y la producción de ruido se atribuye en gran parte a los automotores particulares, de transporte público y de mercadería que transitan en las avenidas principales de la ciudad. Una solución adecuada no podría ser solamente la introducción de áreas verdes y la siembra de árboles en el espacio público sin contemplar la forma de reducir el parque vehicular a través de un sistema de transporte público eficiente para la población. La regulación del clima ante los efectos de la isla de calor debe ser enfrentado con acciones que consideren la recuperación del ecosistema, su desarrollo hacia el interior de la ciudad e ir asegurando que las políticas de suelo y construcción consideren variables térmicas.

El tratamiento de aguas residuales y el tratamiento de desechos sólidos y reciclaje de nutrientes presentan resultados altamente valorizados desde enfoques contrarios. El primero evidencia la falta de infraestructura para el tratamiento de aguas residuales como recurso para utilizar los caudales de las cuencas del Río Seco y Xequijel para recolectar las aguas fuera de la ciudad, dañando el ecosistema y ahorrando un monto que debiera ser considerado cuando la oferta de agua en Quetzaltenango no sea suficiente y se vea obligada a reciclarla. El segundo (tratamiento de desechos sólidos y reciclaje de nutrientes) es una oportunidad de mercados para el reciclaje, demostrando que la gestión adecuada de materia orgánica, papel y vidrio se convierte en una fuente de ingresos para el gobierno municipal o empresas privadas.

El SE de Hábitat y Refugio revela que, durante el desarrollo histórico de la Ciudad de Quetzaltenango, el valor ecosistémico no ha tenido la repercusión suficiente para la toma de decisiones sobre el territorio, pues el bosque se ha fragmentado, los caudales de las cuencas se han contaminado y ecosistemas tan particulares como el pantano de la zona 2 se han reducido para permitir el avance urbano. La valoración a través del escenario hipotético recuerda el valor tangible de esos ecosistemas desde los PNFM, biomasa, reciclaje, disponibilidad de agua, reducción del riesgo a desastres y adaptación al cambio climático.

Por último, el SE de Recreación, medido a través del conocimiento, preferencia y valoración desde la población, muestra resultados como la mínima permanencia de la población en las áreas verdes, los factores sociales y ambientales que limitan las visitas al igual que los accesos y la poca valoración ante la condición de estar dispuestos a pagar para ingresar a las áreas verdes. Lo anterior deja en el resultado final un enfoque hacia formas de entretenimiento más atractivas como la visita a centros comerciales, que es el lugar para recreación más frecuentado por la población según la muestra evaluada. 


\section{Conclusiones}

Esta investigación es un intento para evaluar 7 servicios ecosistémicos en la ciudad de Quetzaltenango. La metodología propuesta es un acercamiento para lograr el análisis del impacto ambiental, social y económico que genera la carencia de los SE en el contexto actual y ser presentado de forma clara y sencilla a los tomadores de decisiones y población en general. La forma de evaluación puede fortalecerse con investigaciones posteriores en estudios de los SE por individual. La aproximación económica no debe considerarse como la única forma de evaluar los SE, aunque en este estudio se empleó de manera exploratoria para conocer el impacto frente a una ciudad que continúa su crecimiento demográfico.

Los resultados evidencian un deterioro de los SE por una falta de prioridad, desconocimiento, necesidad de suelo y condiciones económicas ante un crecimiento urbano descontrolado. Si bien las condiciones del valle de Quetzaltenango son de un paisaje con disponibilidad del medio natural suficiente para la población, este ha sido fragmentado y expulsado del área urbana donde reduce su alcance de servicios hacia el interior.

Considerar los servicios ecosistémicos en las decisiones sobre el paisaje urbano es fundamental no solo para la conservación de ecosistemas, sino también para su desarrollo dentro de la ciudad en proporción al medio construido (Register, 2006). Las políticas enfocadas a la calidad ambiental de la ciudad deben ser elaboradas y puestas en práctica como limitantes de la capacidad soporte del territorio, el cual también debe ser objeto de estudio.

Por último, se hace énfasis en la importancia del conocimiento, monitoreo y medición constante de los SE para garantizar la sostenibilidad y bienestar de la sociedad dentro de una región definida. Hace más de mil años en este mismo territorio, los mayas experimentaban un período de decadencia dada por la pérdida de biodiversidad y bosques, degradación del suelo y densificación poblacional que los llevó al colapso como civilización y a una reorganización obligatoria enfocada en resiliencia (Heckbert, Constanza \& Parrott, 2014).

\section{Referencias bibliográficas}

Adedipe, N.O., Bajar, J., Krogmann, U. \& Sridhar, M.K.C. (2005). Waste management, processing, and detoxification Millenium Ecosystem Assessment. Vol. 3. Policy Responses Working Group. Chicago: Island Press.

Akbari, H., Kurn, D., Bretz, S. \& Hanford, J. (1997). Peak power and cooling energy saving of shade trees. Energy and Buildings, 15(2), 139-148.

Baral, H., Guariguata, M. \& Keenan, R. (2016). A proposed framework for assessing ecosystem goods and services from planted forests. Ecosystem Services, 22, 260-268.

Beier, C. \& Gundersen, P. (1989). Atmospheric deposition on the edge of a spruce forest in Denmark. Environment Pollution, 60, 257-271.

Banco Interamericano de Desarrollo [BID]. (2014). Plan de Acción. Xelajú Sostenible. Municipalidad de Quetzaltenango, Guatemala. Recuperado de: https://webimages. iadb.org/PDF/Plan+de+Acción+ICES+Quetzaltenango.pdf

Bolund, P. \& Hunhammar, S. (1999). Ecosystem services in urban áreas. Ecological Economics, 29, 293-301.

Campos, A., Cabrera, P., Pére, C. \& Laura, C. (2017). Tendencia del mercado y a producción de los productos orgánicos en el Perú. Revista Investigaciones Altoandinas, 19(4), 427-431.

Carreras, C., Marin, M., Vide, J., Moreno, M. \& Sabi, J. (1990). Modificaciones térmicas en las ciudades. Avance sobre la isla de calor en Barcelona. Documents D'Analisi Geografica, 17, 51-77. 
Carreño, L., Frank., F. \& Viglizzo, E. (2012). Tradeoffs between economic and ecosystem services in Argentina during 50 years of land-use change. Agriculture, Ecosystems and Environment, 154, 68-77.

Castillo, F., García, J., López, A. \& Celada, M. (2013). Los servicios ecosistémicos en las ciudades de Quetzaltenango y la Antigua Guatemala (Proyecto DIGI 2.26). Universidad de San Carlos de Guatemala. Recuperado de: https://digi.usac.edu. gt/bvirtual/informes/informes2012/INF-2012-36.pdf

Constanza, R., d'Arge, R., de Groot, R., Farber, S., Grasso, M., Hannon, B., Limburg, K., Naeem, S., O'Neil, R., Paruelo, J., Raskin, R., Sutton, P. \& van den Belt, M. (1997). The value of the world's ecosystem services and natural capital. Nature, 387, 253-260.

Constanza, R., de Groot, R., Braat, L., Kubisewski, I., Fioramonti, L., Sutton, S., Farber S. \& Grasso, M. (2017). Twenty years of ecosystem services: How far have we come and how far do we still need to go. Ecosystem Services, 28, 1-16.

Constanza, R., de Groot, Sutton, P., van der Ploeg, S., Anderson, S., Kubiszewski, I., Farber Stephen \& Turner, K. (2014). Changes in the global value of ecosystem services. Global Environmental Change, 26, 152-158.

Cortinovis, C. \& Geneletti, Davide. (2018). Ecosystem services in urban areas: What is there, and what is still needed for better decisions. Land Use Policy, 70, 298-312

El-Baghdadi, O. \& Desha, C. (2016). Conceptualising a biophilic services model for urban areas. Urban Forestry \& Urban Greening, 27, 399-408

Elmqvist, T., Setäla, H., Handel, SN., van der Ploeg, S., Aronson, J., Blignaut, JN., Gómez-Baggethun, E., Nowak, DK., Kronenberg, J. \& de Groot, R. (2015). Benefits of restoring ecosystem services in urban áreas. Current Opinion in Environmental Sustainability, 14, 101-108

Environmental Protection Agency [EPA]. (2018, marzo). Greenhouse gas emissions from a typical passenger vehicle. Ann Arbor. Recuperado de: https://www.epa. gov/greenvehicles/greenhouse-gas-emissions-typical-passenger-vehicle

Escobar, J. (2002). La contaminación de los ríos y sus efectos en las áreas costeras y el mar (Serie 50). Comisión Económica para América Latina CEPAL. Recuperado de: https://archivo.cepal.org/pdfs/Waterguide/LCL1799S.PDF

Ewel, K. (1997). Water quality improvement by wetlands. Nature services. Societal Dependence on Natural Ecosystems. Washington: Island Press.

García, L., Curetty, G., Garegnani, G., Grilli, G., Pastorella, F. \& Paletto, A. (2016). La valoración de los servicios ecosistémicos en los ecosistemas forestales: un caso de estudio en los Alpes Italianos. Bosque, 37(1), 41-52.

Haines-Young,R.\&Potschin,M.(2018).CommonInternationalClassificationofEcosystem Services and guidance of the application of the revised structure. Recuperado de: https://cices.eu/content/uploads/sites/8/2018/01/Guidance-V51-01012018.pdf

Heckbert, S., Constanza, R. \& Parrott, L. (2014). Achieving sustainable societies: Lessons from modelling the Ancient Maya. Solutions Journal, 5(5), 55-64.

Hough, M. (2004). Cities \& Natural Process. A basis for sustainability. New York: Routledge.

Ingram, J.C., Wilkie, D., Clements, T., Mcnab, R. B., Nelson, F., Baur, E. H. \& Foley, C.A.H (2014). Evidence of payments for ecosystem services as a mechanism for supporting biodiversity conservation and rural livelihoods. Ecosystem Services, 7(2014), 10-21

Instituto de Investigación y Proyección sobre Ambiente Natural y Sociedad de la Universidad Rafael Landívar [IARNA-URL]. (2018). Ecosistemas de Guatemala basado en el sistema de clasificación de zonas de vida (Documento IARNA 42). Recuperado de: http://www.infoiarna.org.gt/wp-content/uploads/2019/02/ Ecosistemas-de-Guatemala-final.pdf

Instituto Geográfico Nacional [IGN]. (2017). Archivos geográficos digitales de Guatemala. Guatemala. 
Instituto Nacional de Sismología, Vulcanología, Meteorología e Hidrología [INSIVUMEH]. (2019). Notas climáticas Quetzaltenango. Guatemala. Recuperado de: http://www. insivumeh.gob.gt/normas-climaticas/

Lara, A., Little, C., Urrutia, R., McPhee, J., Álvarez-Garretón, C., Oyarzún, C., Soto, D., Donoso, P., Nahuelhual, L., Pino, M. \& Arismendi, I. (2009). Assessment of ecosystem services as an opportunity for the conservation and management of native forests in Chile. Forest Ecology and Management, 258, 415-424.

Lara-Pulido, J., Guevara-Sanguinés, A. \& Arias, C. (2018). A meta-analysis of economic valuation of ecosystem services in Mexico. Ecosystem Services, 31, 126-141.

Lo, V. (2016). Synthesis report on experiences with ecosystem-based approaches to climate change adaptation and disaster risk reduction (Technical Series No.85). Recuperado de: https://www.cbd.int/doc/publications/cbd-ts-85-en.pdf

López, M. (2019). The informalities of formal settlements in Guatemala. Shelter Policy Review. Hudco Shelter Review, 20(1). 85-95.

López, M., Carrasco, C. \& Valenzuela, A. (2017). Propuesta simbiótica natural-cultural en territorio Mapuche de Arauco. Investigaciones Geográficas, 54, 61-84.

Margulis, L. (2002). Planeta simbiótico. Un nuevo punto de vista sobre la evolución. Barcelona: Debate. Martínez, G., Oaxaca, J. \& Guerra, R. (2011). Productos Orgánicos; Agronegocio existoso en México. Revista Mexicana de Agronegocios, 15, 503-513.

McHarg, I. (1967). Design with nature. New York: The National History Press.

Millenium Ecosystem Assessment [MEA]. (2005). Ecosystems and human well-being: Synthesis. Washington D.C.: Island Press.

Morán, A. (2018). La metrópoli de Los Altos: La expansión desordenada. Revista Análisis de la Realidad Nacional, 24(7), 129-142.

Mumford, L. (1961). The city in history: its origins, its transformations and its prospects. New York: Harcourt.

Municipalidad de Quetzaltenango. (2017). Plan de Ordenamiento Territorial. Quetzaltenango. Recuperado de: https://www.scribd.com/document/355062568/ Plan-de-Ordenamiento-Territorial-Del-Municipio-de-Quetzaltenango-2017Compressed

Murti, R. \& Buyck, C. (Ed.). (2014). Safe Heavens: Protected areas for disaster risk reduction and climate change adaptation. Gland Switerland: IUCN.

Organización Mundial de la Salud [OMS]. (2015). 1100 millones de personas corren el riesgo de sufrir pérdida de audición. Ginebra. Recuperado en: https://www.who. int/mediacentre/news/releases/2015/ear-care/es/

Pagiola, S. (2008). Payments for environmental services in Costa Rica. Ecological Economics, 65(4), 712-724.

Pagiola, S., Zhang, W. \& Colom, A. (2010). Can payments for watershed services help save biodiversity? A spatial analysis of highland Guatemala. Journal of Natural Resources Policy Research, 2(1), 7-24.

Posada, M., Arroyave, M. \& Fernández, C. (2009). Influencia de la vegetación en los niveles de ruido urbano. Revista EIA, (12), 79-89.

Register, R. (2006). EcoCities: Rebuilding cities in balance with nature. Gabriola Island: New Society Publishers.

Reynolds, K. (2002, septiembre/octubre). Tratamiento de aguas residuales en Latinoamérica. Identificación del Problema. De la llave. Recuperado en: http:// cidta.usal.es/residuales/ibros/documentos_nuevos/DeLaLaveSepOct02.pdf

Ricaurte, L., Olaya-Rodríguez, M., Cepeda-Valencia, J., Lara, D., Arroyave-Suárez, J., Finlayson, C. \& Palomo, I. (2017). Future impacts of drivers change on wetland ecosystem services in Colombia. Global Environmental Change, 44, 158-169.

Rogers, R. \& Gumuchdjian, Ph. (2000). Cities for a small planet. Boulder: Westview Print. 
Ruiz, C., Cardona, D. \& Duque, J. (2012). Corredores Biológicos, una estrategia de recuperación de paisajes altamente fragmentados. Gestión y Ambiente, 15(1), 7-18.

Russo, A. \& Cirella, G. (2017). Biophilic Cities: planning for sustainable and smart urban environments. En R. Aijaz (Ed.), Smart cities movement in BRICS (pp. 153-159). New Delhi: Vincent Advertising.

Superintendencia de Administración Tributaria [SAT]. (2019, junio). Parque vehicular de Quetzaltenango. Guatemala. Recuperado de: https://portal.sat.gob.gt/portal/ descarga/1741/estadisticas-tributarias/5404/pvdepartamento.xlsx

Sistema Integrado de Administración Financiera Municipal [SIAF-MUNI]. (2018). Reporte de Gastos por Función. Guatemala. Recuperado de: http://siafmuni.minfin.gob.gt/ siafmuni/GASTOSnew.aspx?pResolucion=1024

Svensson, M. \& Eliasson, I. (1997). Grönktrukturens betydelse för stadens ventilation. Naturvärdsvekets. Stockholm: Swedish Environmental Protection Agency.

Szota, C., Coutts, A., Thom, J., Virahsawmy, H., Fletcher, T. \& Livesley, S. (2019). Street tree stormwater control measures can reduce runoff but may not benefit established trees. Landscape and Urban Planning, 182, 144-155.

Tacam, J. (2011). Modelo conceptual del acuífero libre del Valle de Quetzaltenango, Ciudad de Quetzaltenango, Guatemala (Tesis de Maestría). Universidad del Valle, Colombia.

The Economics of Ecosystems and Biodiversity [TEEB]. (2010). Mainstreaming the Economics of Nature: A synthesis of the approach, conclusions and recommendations of TEEB. Recuperado de:http://www.biodiversity.ru/programs/ international/teeb/materials_teeb/TEEB_SynthReport_English.pdf

Thompson, C., Roe, J., Aspinall, P., Mitchell, R., Clow, A. \& Miller, D. (2012). More green space is linked to less stress in deprived communities: evidence from salivary cortisol patterns. Landscape and Urban Planning, 105(3), 221-229.

United Nations [UN]. (2018, 16 de mayo). $68 \%$ of the world population projected to live in urban areas by 2,050 says UN. New York. Recuperado de: https://www.un.org/ development/desa/en/news/population/2018-revision-of-world-urbanizationprospects.html

Agencia de los Estados Unidos para el Desarrollo Internacional [USAID]. (2017). Diagnóstico de la situación de la generación y manejo de los desechos sólidos en los municipios de la Mancomunidad Metrópoli de los Altos. Recuperado de: https:// nexoslocales.com/wp-content/uploads/2017/12/Diagn\%C3\%B3stico-Metropolide-los-Altos-CA-25-de-agosto-2017_sb.pdf

Viera, F. (2003). Geomorphology and natural hazards of the Samala river basin, Guatemala (Tesis de Maestría). International Institute for Geo-Information Science and Earth Observation, The Netherlands.

Yang, L., Quian, F., Song, D. \& Zheng, K. (2016). Research on Urban Heat-Island Effect. $4^{\text {th }}$ International Conference on Countermeasures to Urban Island Effect UHI 2016. Procedia Engineering, 169, 11-18.

Yeang, K. (2002). Reinventing the skyscraper: A vertical theory of urban design. Chinchester: Willey Academy. 